\title{
Self-propelled autonomous 2D parallel robots for sliding cupping-glass massage for back and chest
}

\author{
Sergey Sayapin ${ }^{1, *}$, Elena Sayapina ${ }^{2}$ \\ ${ }^{1}$ Institute for Machine Science named after A.A. Blagonravov of the Russian Academy of Science, 101990 Moscow, Russia \\ ${ }^{2}$ A. Tsyb Medical Radiological Research Centre, 249036, Obninsk, Russia
}

\begin{abstract}
There are numerous examples of effective using of sliding cupping-glass massage for disease prevention, treatment, rehabilitation, and removal of weariness. This is kind of massage including combination influences to patient's back and chest by cupping-glasses and massage. However, work of masseur is connected with a big physical stress. Moreover, masseur can make the massage only with one patient by two hands. As a result daily productivity of the masseur is limited. Therefore advanced intelligence carried massage robots are needed for sliding cupping-glass massage, being able to work autonomously without the presence of the masseur. In this paper, a novel approach to a creation of intelligence self-propelled autonomous portable massage robot based on original 2D parallel mechanism is presented. This robot based on triangular parallel structure with 3 d.o.f., called the Triangle, is designed for sliding cupping-glass massage for back and chest. Main massage manipulations of robot are presented along with a description of proposed locomotion algorithms. Locomotion and manipulation experiments are performed by pneumatic prototypes. We examine design principles of the Triangle as future intelligent base block for various rehabilitation and other robotic systems that can self-propelled and self-reconfigure.
\end{abstract}

\section{Introduction}

It is known that a massage is now considered one of the most effective non-drug methods of effective means of disease prevention, treatment, rehabilitation, removal of weariness. Therefore now massage is used in clinics, hospitals, and medical centers of many countries. All kinds of the massage are made by a masseur or a patient (self-massage) manually or by massage apparatuses [1]. In order to augment an effect of traditional manual techniques, the masseur may use other massage techniques, for example, such as a sliding cupping-glass massage (SCGM) for back and chest. However, usually SCGM is independent kind of manual massage including combination influences to patient's back and chest by the cupping-glasses and massage. There are numerous examples of effective using of SCGM in practice medicine [1]. In Fig. 1 (a) basic massage movements for SCGM by vacuum massage cupping glass (VMCG) are shown. These movements include following: I - straight, II - zigzag, III - spiral, and IV - figure-of-eight. The masseur makes the massage personally. Therefore the presence of the masseur is required, but he can make the massage only with one patient by two hands. As a result a daily productivity of the masseur is limited. Moreover, the masseur usually performs laborious and monotonous manipulations all day long that lead to his fatigue. A work of the masseur is connected with a big physical stress that can be cause of pathological changes in spine and lumbar one. The self-massage also demands from patient of the big physical stress that is inadmissible for separate categories of patients. Therefore labor-intensive procedures of the massage should be robotized.
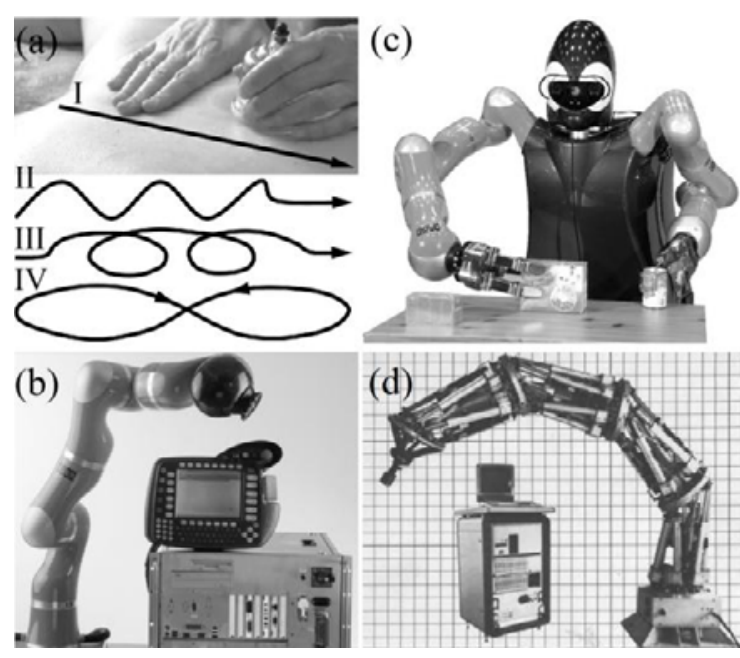

Fig. 1. Basic massage movements by VMCG (a); the KUKA lightweight robot with controller (b); mobile dual arm robot with 3-finger hands (c); the multi-module parallel robot Logabex LX4 (d).

Currently, there are variable robots, which can be used as base of half-autonomous or autonomous massage robots, for example, such as anthropomorphic one/twoarmed robots (similar to the KUKA lightweight robot) [2] or multi-module parallel robots (similar to the 
Logabex robot LX4) [3], which are shown in Fig. 1 (b, c, and d). Unfortunately, now there are only few examples of practical use of anthropomorphic one-armed massage robots which was created similar to the industrial robots [4]-[6]. However, these one-armed robots have large sizes and weight, and therefore, they cannot be used in carried massage robotic systems. Furthermore, in case of the patient's body movements, the programmed motion of massage robot is not suitable. Thus, a creation of the carried massage robots for SCGM for back and chest being able to work autonomously without the presence of the masseur is an urgent task. Therefore modern rehabilitation medicine is needed of advanced intelligence carried massage robots, being able to work autonomously without the presence of the masseur. I propose new concept of a self-propelled multifunctional intelligence autonomous carried planar massage robot for SCGM for back and chest based on a triangular parallel structure with 3 d.o.f., called the Triangle [7]. The description of design principles of the Triangle as future intelligence base block for various rehabilitation and other robotic systems that can self-propelled and self-reconfigure is presented below.

\section{Description of Triangle}

The Triangle is based on the 2D triangular parallel mechanism and therefore it has higher a specific stiffness and a bearing capacity, and lower specific weight. In Fig. 2 a structural scheme of the Triangle is shown. The proposed concept will allow be robotize the process of SCGM. As a result there are the following advantages:

- The Triangle is able to organize autonomous conduction of SCGM to several patients simultaneously, and holding cupping massage by several vacuum massage cupping-glasses (VMCG) in the sitting or standing, for example by three VMCG on chest and three VMCG on back of patient at the same time and, as a result the daily productivity of the masseur increases.

- There is massage by sliding and stretching of muscle tissues at the same time the three VMCG that improves the performance of the process of massage.

- There is the autonomous SCGM to hard reached areas of the body such as back, and ensure the controlled movement of VMCG and VMCG bypass the moving parts of body that are not valid for massage effects, such as moles, sensitive and damaged skin, post-operative wounds, etc.

- The autonomous SCGM does not require special facilities and can be performed at the bedside and at the home.

- The Triangle released extra time, which can be used to conduct other types of massage and, as a consequence, there is a possibility of increasing the number of patients served per shift.

- There is the reduction of the physical fatigue of the masseur, thereby increasing his productivity and improving health at the end of the work shift.

The Triangle (Fig. 2) has a massage device based on the active triangular module $\mathrm{ABC}$ with the parallel structure. The sides $\mathrm{AB}, \mathrm{BC}$ and $\mathrm{CA}$ are made in the form of similar rods. The ends of rods are pivotally connected at the vertices of the active triangular module $\mathrm{ABC}$ with the VMCG 1. Thus, each of the rods is provided a linear drive 2 , a force sensor 3 , a relativedisplacement sensor 4, and a relative-velocity sensor 5 . All vertices of triangle module $\mathrm{ABC}$ are executed with possibility of connection the like rods through them 2 for the formation of the additional active triangular parallel structures. Each of VMCG 1 is pivotally connected at the vertices of the active triangular module $\mathrm{ABC}$ and forms its device discrete fixation to the patient's body. In addition, each of the VMCG 1 is hermetically connected by a flexible hose 6 to a mated canal of an air distributor 7 of a degassing system provided with a pressure sensor 8 , the air distributor 7 and a vacuum pump 9 electrically connected to a control system 10 in the form of a neural computer 11 with digital-to-analogue converters (DAC), and relevant software (the main advantages of neural computer are associated with massively parallel processing, and as the result is high speed, low requirements for stability and accuracy of the parameters of the elementary nodes, resistance to interference and destruction). The inputs of the control system 10 are connected through the data buses of analog-digital converters (ADC) to the outputs: of ADC 12, 13, 14 and 15 of the force sensors 3 for ADC 12; the relativedisplacement sensors 4 for ADC 13; the relative-velocity sensors 5 for ADC 14; and the pressure sensors 8 for ADC 15. The outputs of the control system 10 are connected through the output data buses to the corresponding inputs of software and the following units, connected in series: DAC 16, power amplifiers 17, linear drives 2, air distributor 7, and vacuum pump 9. The control system 10 made with the possibility of operational control and management in real-time.

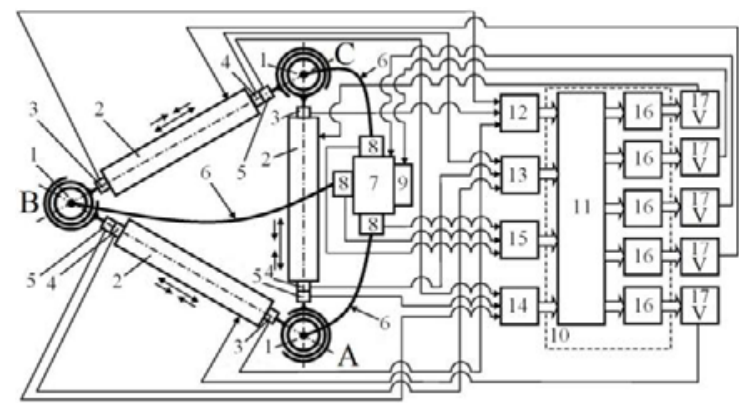

Fig. 2. Structural scheme of Triangle.

The Triangle works as follows. On surface of the patient's body [1] an active triangle module $\mathrm{ABC}$ applied with VMCG 1 in its vertices (Fig. 3, a, b). The initial position of the active triangle module $\mathrm{ABC}$ is mounted on the massaged area of the patient's body arbitrary. Then the VMCG 1 degas by the vacuum pump 9 (Fig. 2) and after their individual suction and retraction of the skin on the value for reliable fixation of VMCG 1 and excluding injury of the patient's skin, which is determined visually, close the corresponding valve (in the figures not shown) of the air distributor 7 and record the value of the minimum allowable pressure with pressure sensors 8 . Then, using the appropriate valves 
(on the pictures conventionally are not shown) of the air distributor 7 is produced depressurization of VMCG 1 to the maximum pressure inside them, which ensures the possibility of their sliding mobility according to the patient's body without detachment from him. At this point, these valves of the air distributor 7 and with the help of pressure sensors 8 are fixed values, the maximum allowable pressures inside the VMCG 1. Further, control system 10 enters data, which include the contours of body areas massaged, associated with the base coordinate system and the coordinates of the areas of the body that are not valid for massage effects, such as moles, sensitive and damaged skin, surgical wound, etc. Then the control system 10 gives the command to the vacuum pump 9 and the air distributor 7 to degas through the flexible hoses 6 and the respective valves of the air distributor 7 two fixed VMCG 1 on surface of the patient's body and one sliding VMCG. After reaching in these fixed and sliding VMCG the minimum allowable and maximum allowable pressures from the respective pressure sensors 8 through the ADC 15 receives analog signals into the control system 10 in which the control commands are formed, which through the corresponding DAC 16 and power amplifier 17 are fed to the closure of the valves of the air distributor 7 and the shutdown of the vacuum pump 9. After that, relative to the base coordinate system entering coordinates of the centers of the two fixed VMCG and one sliding VMCG and the distance between them. Then, the masseur gives a command to perform the programmed massage movements for the sliding VMCG.

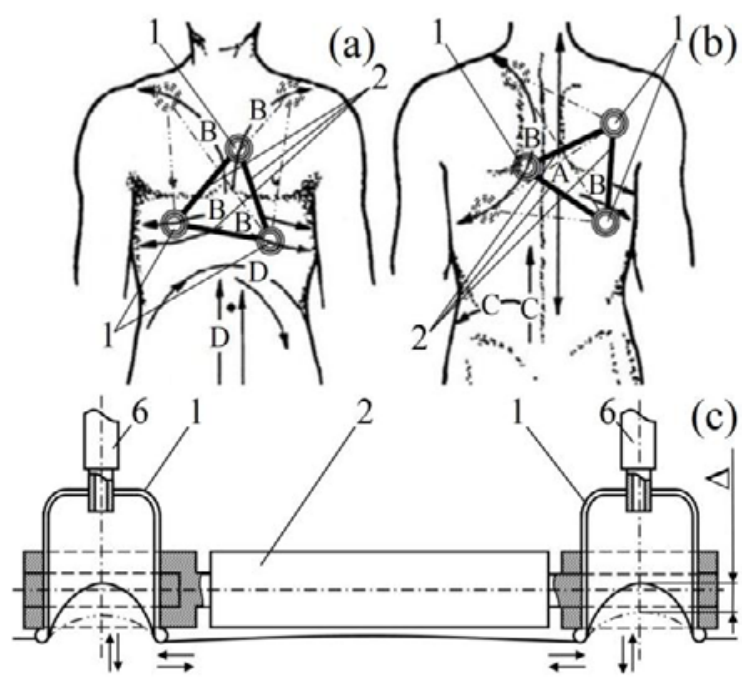

Fig. 3. Structural scheme of Triangle.

In Fig. 3 (a, b) schemes of the SCGM in various diseases are shown: spinal osteochondrosis and lumbago (A); pneumonia and bronchitis (B); myositis and sciatica (C); colitis and hypertension (D) [1]. As we can see in the above diseases impose the straight movements of the one sliding VMCG 1 and its movements along the circular arcs. After receipt of the command to the control system 10 in the computer 11 (taking into account the previously entered data) the initial coordinates of the VMCG 1 for carrying out massage movements of the
VMCG 1 are calculating, for example arcuate movements (B) (Fig. 3, a, b), from the condition of ensuring a minimum number of VMCG rearrangements required to convert the selected path of movement, which depend on the maximum strokes of the linear drives 2. The active triangle module $\mathrm{ABC}$ can be installed in the initial position in such way that each of his VMCG 1 will be able to alternately perform the rolling massage movement on different trajectories of the movements of $b$ without its permutations (Fig. 3, b). The movement of the VMCG 1 on the surface of the patient's body is carried out by appropriately changing the lengths of the linear drives 2, controlled relativedisplacement sensor 4. Thus the required speed of the massage movements of the VMCG 1 on the patient's body is provided by the speed control of axial movements of the linear drives 2 according to signals from the respective relative-velocity sensors 5 . The geometric resistance of the active triangular module $\mathrm{ABC}$ (Fig. 3) allows you to define the coordinates of its vertices $\mathrm{A}, \mathrm{B}$ and $\mathrm{C}$ by measuring by the relativedisplacement sensor 4 of the lengths of all rods and control their movements similarly to the organization of spatial movements of the $\boldsymbol{l}$-coordinate manipulator [8]. The relative-velocity sensor 5 allow to control the speed of movement of the sliding VMCG 1 at this stage of massage in accordance with the valid values, defined for this type of massage movements and introduced to the control system 10 . It should be noted that all of the VMCG 1 in the course of their rearrangements through sliding motions on the massaged surface of the patient body, alternately become floating and unmoveable. Here in, the process of their permutation. In the active triangular module $\mathrm{ABC}$ one of the VMCG 1 closest to his move) and the other two are roaming and unmoveable, respectively. While the maximum allowable air pressure sets in one roaming sliding VMCG, and in two fixed VMCG - minimum allowable air pressure. After that, the control system is commanded to switch on the linear drives 2 , is connected to the sliding VMCG 1 and produced a consistent change in their lengths at a given speed controlled by the relativespeed sensor 5. After moving the sliding VMCG 1 to a specified destination (or intermediate) point, determined by the relative-displacement sensor 4 associated with linear drive 2, the command is made to switch them off. Then displacement of the sliding VMCG becoming the fixed VMCG, and one of the two fixed VMCG is becoming roaming. The pressure in the displaced VMCG is the minimum, and another is allowable maximum. Further, similar to the previous cycle from the control system is commanded to switch on the linear drive 2 , is connected to the moving VMCG and produced a consistent change in their lengths at a given speed controlled by the relative-speed sensor 5 . Then similarly do the movement of third VMCG in final or intermediate point. After displacement of all VMCG in the calculation the initial coordinates two of the VMCG are made unmoveable, and the third lying on the trajectory massage movements, for example the arc B (Fig. 2, b), make sliding cupping-glass and make her massage movements. Thus, depending on the desired massage 
motions, the estimated initial coordinates of VMCG can be computed in such way that all VMCG will be on the trajectories required of massage movements, for example on the arcs $\mathbf{B}$ (Fig. 3, b). In this case, the massage can be performed sequentially and alternately. Permutations of triangular $\mathrm{ABC}$ module can be arranged in such way that the trajectory of all movements VMCG will coincide with the specified trajectory of massage movements in the process of permutations, i.e. there will not be "spurious" passages of VMCG.

Using the Triangle it's also able to produce a local vacuum massage and the massage by sliding and stretching of the muscle tissue [1]. In Fig. 3 (c) the scheme of their conduct is shown. Local vacuum massage is performed at a fixed on the patient's body VMCG 1 by the vacuum in the air up to a pressure whose value is in the range from the maximum pressure to the minimum pressure. For its implementation by commands from the control system in the VMCG with established frequency and amplitude of the pressure change from the minimum permissible to the maximum and Vice versa. Management of alternate higher and lower pressure is carried out using the diffuser 7 and vacuum pump 9 according to the commands from the control system 10, formed as a result of processing the signals from the pressure sensors 8 . The distance between the involved skin surface at the maximum pressure inside the jar and the involved skin surface with minimal pressure is $\Delta$ (Fig. 3). The distance $\Delta$ corresponds to the maximum value of the amplitude in the conduct to local vibration by varying the pressure in the VMCG.

Massage by sliding and stretching the muscle tissues is produced, while still fixed on the patient's body VMCG 1 by discharging air to the minimum allowable pressure. It is possible to carry out this type of massage as alternate movement of the linear actuators, as the simultaneous movement of two or more linear actuators. After fixing the VMCG 1 on the massaged area of the body of the patient (Fig. 3) at the position at which the length of the linear actuator 2 correspond to their average values, the control system 10 is commanded to the linear drive 2 on decrease (increase) its length at a predetermined value, after reaching which the linear actuator 2 is stopped and is switched on for reverse, increasing (decreasing) its length to a set value. The process is repeated at a given rate, the required number of cycles. The maximum change in length of the linear actuator 2 corresponds to a given vibration amplitude of the massage movements of compression-tension. Changing the length of the linear actuator 2 and the amplitude are controlled by the relative movement of the sensor 4 and the speed - with the help of relative velocity sensor 5 . The efforts of the mechanical effects of VMCG 1 on the muscle tissue of the patient performing all types of massage are controlled by the force sensor 3. In case of exceeding the set value of the effort in conducting a moving cupping massage, on command from the control system 10 produces an increase in pressure in the VMCG 1 by the amount of the established step and take him for the maximum allowed pressure value and continue massage. In case of exceeding a predetermined force value during the massage by sliding and stretching of muscle tissue, control system 10 reduces the magnitude of the amplitude which is set by the permissible forces in the extreme positions of the linear actuator 2 and continue the massage.

In case of spontaneous detachment of one of vacuum massage cupping-glasses from the patient's body during the massage unplanned increase in her pressure happens, as in the control system 10 receives the corresponding signal from the pressure sensor 8 . In response to the situation the control system 10 will give the appropriate command to the distributor valve 7 and vacuum pump 9 . It should be noted that the active triangular bearing module $\mathrm{ABC}$ on the massaged area of the body of the patient occurs at three points in the form of VMCG 1 . Therefore, in the case of the horizontal position of the patient gravity from $1 / 3$ the weight of the active triangle module $\mathrm{ABC}$ will be enough for a snug fit to the surface of the seal body VMCG 1 and subsequent suction to the body after lowering the pressure to the minimum allowable value. This will mean that the VMCG 1 is recorded on the patient's body and the massage will continue in accordance with the established program. In case of the pressure decrease in the cupping-glass is not going to happen, from the control system 10 will receive the signal about the movement of the VMCG to a new location and repeat the procedure vacuum. And, in case of repeated unsuccessful attempts, from the control system 10 the massage therapist will receive the signal about emergency situation, for correction of which requires his involvement. After the liquidation of the emergency situation massage therapist, the massage procedure will be continued offline.

We implemented the Triangle concept with a real pneumatic prototype that uses nine syringes $(60.0 \mathrm{ml})$ and three barrels $(60.0 \mathrm{ml})$ as three linear pneumatic actuators with three pneumatic pumps and as three VMCG with three vacuum pumps (Fig. 4, a). The pneumatic prototype is operated manually.

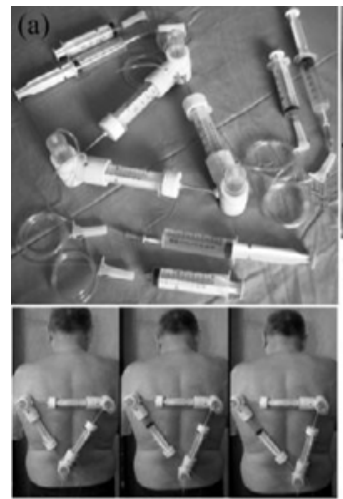

(b)

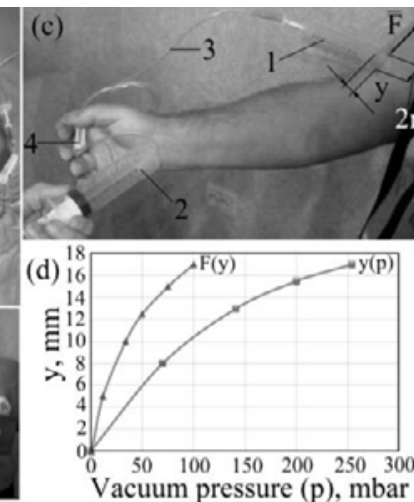

Moving force $(\mathrm{F}), \mathrm{N}$
Fig. 4. The pneumatic prototype of Triangle (a); SCGM of the back by the pneumatic prototype of Triangle (b); the prototype of VMCG (c); the experimental relationships between (y) and $(\mathrm{p})$, and (F) and (y) for the prototype of VMCG (d).

We also performed locomotion experiments and SCGM of the patient's back by the pneumatic prototype 
(Fig. 4, b). In Fig. 4 (c) a prototype of VMCG is shown, which consists of one barrel 1 , one syringe 2 , and tubing 3 with a roller clamp 4 of an infusion system. This prototype is the same as VMCG with vacuum pump of the real pneumatic prototype (Fig. 4, a, b). Fig. 4 (d) illustrates the experimental relationships between skin uplift (y) and a vacuum pressure (p), and also between skin uplift (y) and moving force (F). Fig. 4 (d) also illustrates the relationship between moving force $(\mathrm{F})$ and vacuum pressure (p). In the experiments, vacuum pressure loadings applied using the prototype of VMCG was subjected to the male's shoulder skin in the 60 year age range. The experiments were conducted by gently pressing barrel 1 of diameter $29.0 \mathrm{~mm}$ against the skin to ensure a good initial contact and then activating syringe 2 (vacuum pump) to create the vacuum inside the barrel of syringe 2 so that the skin is drawn into the barrel 1 (Fig. $4, \mathrm{c}$ ). The bulging of the skin as a result of cupping is shown in Fig. 4; all images were captured using a Canon digital camera IXUS 990 15. Moving forces (F) were measured using the Dynamometer 5029 DPU-0.1-2 (GOST 13837-79). The vacuum pressures (p) developed inside the prototype of VMCG were calculated using Boyle's Law:

$$
p_{\mathrm{i}} V_{\mathrm{i}}=p_{\mathrm{f}} V_{\mathrm{f}},
$$

where the subscripts $\mathrm{i}$ and $\mathrm{f}$ refer to initial (before pumping, $p_{\mathrm{i}}=1000 \mathrm{mbar}$ ) and final (after pumping) states and measured volumes of barrel 1 (prototype of VMCG, $60.0 \mathrm{ml})$ with tubing $3(3.18 \mathrm{ml})$ and barrel $(60.0 \mathrm{ml})$ of syringe 2 (vacuum pumping chamber) which is decreased by volume of skin uplift $\left(V_{\text {su }}\right)$. The volumes of skin uplift (Fig. 4, c) were calculated as volumes of ball segment $\left(V_{\mathrm{su}}=\pi y^{2}(3 r-y) / 3\right)$.

\section{Other kinds of locomotion of Triangle}

The Triangle can locomote also without vacuum pressure using other types of locomotion, for example, such as a leopard crawl (Fig. 5, a), a vertical rolling (similar to the tetrahedral walker movement) [9], and a shuffle (Fig. 6). The leopard crawl (Fig. 5, a) can be used when locomotion of the Triangle on a horizontal or inclined surface.

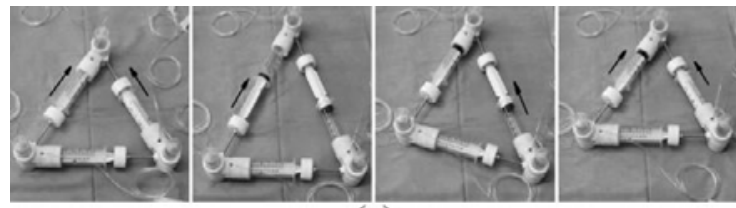

(a)

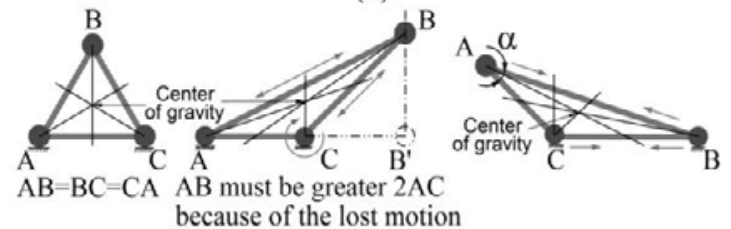

(b)

Fig. 5. Locomotion of the Triangle by using the leopard crawl (a) and the vertical rolling (b).
The Triangle can shuffle one's feet when moving in a vertical plane as shown in Fig. 6. The movement cycle of the Triangle includes following stages: initial position (a), a displacement of the center of gravity left (b), forward translation of right joint by using the shuffle (c), the displacement of the center of gravity right (d), forward translation of left joint by using the shuffle (e), final/initial position (a). Also the pneumatic prototype of the Triangle can be rotated by the plunger installed in right or left prototype of the VMCG (f).

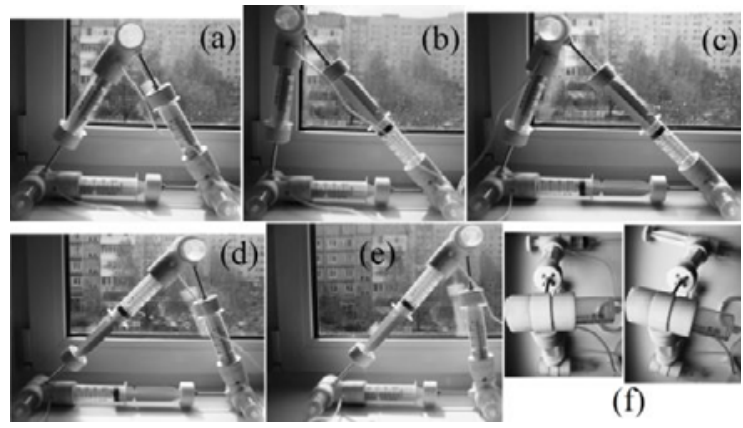

Fig. 6. The movement cycle of the Triangle by using the shuffle (a-e) and the ability of rotation (f).

The locomotion of the Triangle in the vertical plane (Fig. 5, b and Fig. 6) can be used when movement in narrow aisles, for example between the walls. The angle $\alpha$ is chosen based on design parameters of the Triangle.

\section{Conclusions}

Any suitable robots for autonomous SCGM haven't found.

We presented the novel approach to a creation of intelligence self-propelled autonomous portable massage robot for SCGM for back and chest based on original planar parallel mechanism. The proposed novel concept of SCGM robot, called the Triangle, can be used in physiotherapy offices, hospitals, rehabilitation, sports and Wellness centers, as well as at home to conduct autonomous self-massage hard to reach areas of the body.

The use of the Triangle will allow you to automate the process of SCGM and to improve its efficiency by increasing the number of simultaneously roaming the VMCG up to three more (with the capacity of moving massager additional linear actuators and the VMCG), as well as significantly increase the number of concurrent patients with a single therapist and reduce his physical weariness and fatigue. Thus it's possible additional installation of sensors at the vertices of a triangular active module $\mathrm{ABC}$, e.g. ultrasound, which will allow you to extend the functionality of the massager moving.

The Triangles could be used as future intelligent base block for various rehabilitation and other robotic systems that can self-propelled and self-reconfigure.

Current objective is to design software and equip the Triangle with reliable and miniature electronics, linear drives and communication hardware.

Another future task is in applying of the Triangle 
towards on-line diagnostics of patient's health during the rehabilitation procedure.

\section{References}

1. V.I. Dubrovsky, A.V. Dubrovskaya, Therapeutic massage. GEOTAR-Media, Moscow (2004)

2. R. Bischoff, J. Kurth, G. Schreiber, R. Koeppe, A. Albu-Schäffer, A. Beyer, O. Eiberger, S. Haddadin, A. Stemmer, G. Grunwald, and G. Hirzinger, The KUKA-DLR Lightweight Robot arm-a new reference platform for robotics research and manufacturing, in: Proceedings of the Robotics (ISR), 2010 41st international symposium on and 2010 6th German conference on robotics (ROBOTIK), pp. 741-748. VDE Verlag GmbH, Berlin (2011)

3. J.-P. Merlet, Parallel Robots. 2nd ed. Springer, Dordrecht (2006)

4. V. Golovin, M. Arkhipov, V. Zhuravlev, Force training for position/force control of massage robots. In: New Trends in Medical and Service Robots, pp. 95-107. Springer Int. Publ., Switzerland (2014)

5. Y. Huang, J. Li, Q. Huang, and C. Liu, Design and control of anthropomorphic BIT soft arms for TCM remedial massage, in: Proceedings of the 2013 IEEE/RSJ International Conference on Intelligent Robots and Systems, Tokyo, Japan, November 3-7, 2013, pp. 1960-1965. IEEE (2013)

6. EMMA, The Robot Therapist. Innovation - The Singapore Magazine of Research and Technology and Education, 15, 1 (2016)

7. S.N. Sajapin, M.S. Sajapina, Russian Patent 2551939, Byull. Izobret., 16 (2015)

8. A.Sh. Koliskor, Stanki i Instrument, 12 (1982)

9. R. Belisle, C.H. Yu, R. Nagpal, Mechanical Design and Locomotion of Modular-Expanding Robots, in: Proceedings of the 2010 IEEE International Conference on Robotics and Automation,Anchorage, USA, May 3-8, 2010, pp. 17-23. IEEE (2010) 